\title{
The dynamics of nematode infections of farmed ruminants
}

\author{
M. G. ROBERTS ${ }^{1}$ and J. A. P. HEESTERBEEK ${ }^{2}$ \\ ${ }^{1}$ AgResearch, Wallaceville Animal Research Centre, P.O. Box 40063, Upper Hutt, New Zealand \\ ${ }^{2}$ Agricultural Mathematics Group (GLW-DLO), P.O. Box 100, 6700 AC Wageningen, The Netherlands
}

(Received 16 June 1994 ; revised 29 September 1994; accepted 29 September 1994)

\begin{abstract}
S U M M A R Y
In this paper the dynamics and control of nematode parasites of farmed ruminants are discussed via a qualitative analysis of a differential equation model. To achieve this a quantity, 'the basic reproduction quotient' $\left(Q_{0}\right)$, whose definition coincides with previous definitions of $R_{0}$ for macroparasites, but extends to models with periodic time-varying transition rates between parasite stages or management interventions, is introduced. This quantity has the usual threshold property: if $Q_{0}$ is less than one the parasite population cannot maintain itself in the host population, and in the long term becomes extinct; but if $Q_{0}$ is greater than one the parasite can invade the host population. An alternative quantity, $\mathscr{R}(E)$, that is often easier to calculate is also introduced, and shown to have the same threshold property. The use of these two quantities in analysing models for the dynamics of nematodes in complex situations is then demonstrated, with reference to the dynamics of mixed parasite species in one host; the effects of breeding host animals for resistance to parasitism; and the development of parasite strains that are resistant to chemotherapy. Five examples are discussed using parameters for the dynamics of nematode infections in sheep, and some statements on control policies are derived.
\end{abstract}

Key words: host-parasite models, macroparasites, nematodes, ruminants.

\section{INTRODUCTION}

The concept of the basic reproduction ratio $\left(R_{0}\right.$, also known as the basic reproductive rate) is central in understanding the dynamics of infectious diseases caused by directly reproducing microparasites (Anderson, 1982; Anderson \& May, 1982, 1991; Diekmann, Heesterbeek \& Metz, 1990). $R_{0}$ was defined by Diekmann et al. (1990) as the expected number of secondary cases produced in a completely susceptible population, by a typical infected individual during its entire period of infectiousness. The other references cited above contain essentially the same definition (see also Dobson \& Grenfell, 1994; Mollison, 1994; Scott \& Smith, 1994). A disease can invade and maintain itself in the host population only if $R_{0}>1$. If $R_{0}<1$ the disease cannot maintain itself and in the long run becomes extinct. This is the threshold theorem of Kermack and McKendrick (Kermack \& McKendrick, 1927; Diekmann et al. 1994), and its use in determining long-term disease dynamics is readily apparent.

The concept of $R_{0}$ for macroparasitic infections, leading to an analogous threshold theorem, has also been introduced (for example Anderson \& May, 1991 ; Roberts, Lawson \& Gemmell, 1987; Roberts \& Grenfell, 1991; Roberts, Smith \& Grenfell, 1994). Individual hosts may be classified as infected with a microparasite or not, due to the rapid proliferation of the infectious agent once established, but the number of macroparasites that constitutes an infection is important, and the threshold theorem must be formulated in terms of the dynamics of the parasite population. Anderson \& May (1991, page 436) for example, define $R_{0}$ for a macroparasite as 'the average number of offspring (or female offspring in the case of a dioecious species) produced throughout the reproductive life-span of a mature parasite that themselves survive to reproductive maturity in the absence of density-dependent constraints on population growth'. It is obvious from this statement that the quantity so defined has the required threshold property for macroparasitic infections.

The definition of $R_{0}$ for infectious diseases contains the word typical, and the difficulty of specifying the typical individual led Diekmann et al. (1990) to examine the concept in a rigorous mathematical setting. These authors were able to define $R_{0}$ as the dominant eigenvalue of an operator (a matrix in many applications) that relates the number of infected hosts in the current generation to the number of hosts in the 'next generation'. The dominant eigenvalue provides a measure of the increase in the number of infected hosts from one generation to the next, after many generations have passed. This mode of definition provides a framework within which $R_{0}$ may be defined and calculated in many complicated situations, including those where contact patterns are heterogeneous or host behaviour is age-structured (for details see also Heesterbeek, 1992).

The dynamics of nematode infections of farmed ruminants have been studied by Roberts \& Grenfell (1991, 1992) using a simple three-equation model. 
Important questions relate to the influence of heterogeneity on the dynamics of these infections, for example: the dynamics of mixed parasite species in one host; the effects of breeding host animals for resistance to parasitism; and the development of parasite strains that are resistant to chemotherapy. To investigate these it is necessary to have a formal definition of a threshold quantity for macroparasites that has analogous properties to those of $R_{0}$ for microparasitic infections. A recent paper by Heesterbeek \& Roberts (1994) has developed such a quantity, which has been tentatively called $Q_{0}$, and which one could call the basic reproduction quotient. The change in terminology is to avoid a fruitless debate on whether this is in fact ' $R_{0}$ for macroparasites' (Roberts, 1994a). What matters is that $Q_{0}$ has the useful threshold property: $Q_{0}>1$ implies that the parasite can invade the host population, and $Q_{0}<1$ implies that the parasite population cannot persist. In fact Heesterbeek \& Roberts (1994) presented three related quantities with this property, resulting from mathematical, biological or practical considerations respectively.

In the present paper the logic leading to the definition of $Q_{0}$ is reviewed in biological terms. The model of Roberts \& Grenfell (1991) is developed to include heterogeneity in the host and parasite populations, and the assembled methodology is used to make statements about the control of parasite populations in ruminants, and the development of parasite strains that are resistant to chemotherapy.

\section{THE MODEL}

A simple model comprising three differential equations was used to describe the dynamics of nematode parasites of ruminants by Roberts \& Grenfell (1991). Two state variables were used: $L$ to represent the density of infective larvae on the pasture, and $A$ to represent the mean number of adult parasites per host.

The rate of change of $L$ was described by an equation similar to

$\frac{d L}{d t}=-(\rho+\beta H) L+q \lambda(r) H A$,

where $\rho$ is the rate at which the density of pasture larvae would decrease in the absence of ruminants, $\beta$ is the rate at which larvae are eaten by ruminants, $\lambda(r)$ is the mean rate at which a single adult parasite produces eggs, $q$ is the probability that an egg develops to become an infective larva and $H$ is the number of host animals/unit area.

The difference between equation (1) and that analysed by Roberts \& Grenfell (1991) is that in the earlier paper $L$ was used for the number of larvae on an area that supports one host animal. In the present paper $L$ has been rescaled by a factor $H$, and $\beta$ by a factor $1 / H$, relative to their values in the earlier paper.

The rate of change of $A$, the mean number of adult parasites/host, was described by the differential equation

$\frac{d A}{d t}=\beta p(r) L-\mu(r) A$,

where $\beta$ is as defined above, $p(r)$ is the probability that a larva, once eaten, develops into an adult parasite, and $\mu(r)$ is the rate of mortality of adult parasites.

The factors $\lambda, p$, and $\mu$, in equations (1) and (2) have been written as functions of $r$, which is used as a measure of the average level of acquired immunity in the host population. This is defined by the equation

$\frac{d r}{d t}=\beta L-\sigma r$

and is therefore the average discounted (over time) cumulative larval challenge experienced by the host population. The parameter $\sigma$ is the rate at which $r$ would decrease in the absence of new larval challenge. The rescaling of $L$ and $\beta$ relative to their usage by Roberts \& Grenfell (1991) does not affect equations (2) and (3).

In the model of Roberts \& Grenfell (1991), $\lambda$ and $p$ may decrease as $r$ increases, and $\mu$ may increase. Our desired threshold quantity, however, is defined in the absence of acquired immunity, that is when $r=0$. There is an inherent contradiction in this, as by equation (3) if a host has experienced infection then $r$ cannot be zero. The threshold is, nevertheless, a limiting quantity that determines the response of a previously parasite-free host-parasite system to the first introduction of the parasite. The level of immunity that has been acquired by the host is, at this time, so small that its effect on the dynamics is negligible.

The rate of production of infective larvae by an adult parasite in the absence of acquired immunity is $q \lambda(0)$, and the average life-span of the adult is $1 / \mu(0)$ years. Hence the average number of larvae due to one adult parasite over its entire life would be $q \lambda(0) / \mu(0)$. Using a similar argument, the average number of adult parasites per host produced by one infective larva in the absence of acquired immunity would be $\beta p(0) /(\rho+\beta H)$. Putting these formulae together, the number of adult parasites that would be produced in the next generation by $A$ adult parasites in the present generation is $Q_{0} A$, where

$Q_{0}=\frac{q \lambda(0)}{\mu(0)} \frac{\beta p(0)}{(\rho+\beta H)} H$.

A quantity equal to $Q_{0}$ (recall our rescaling of $\beta$ ) was described by Roberts \& Grenfell (1991) as the basic reproductive rate of infection ( $=$ the basic repro- 
duction ratio of the parasite population). Those authors showed that if $Q_{0}>1$ then equations (1)-(3) have a unique non-zero steady-state solution for $L, A$ and $r$, whereas if $Q_{0}<1$ all three variables tend to zero with time, corresponding to the parasite population becoming extinct.

The argument leading to the definition of $Q_{0}$ could have been formulated differently, as follows. One adult parasite would, over the course of a year, give rise to $q \lambda(0)$ larvae, each with a life-expectancy of $1 /(\rho+\beta H)$ years; in other words $q \lambda(0) /(\rho+\beta H)$ larva-years. Similarly, each larva-year would, in the absence of acquired immunity give rise to $\beta H p(0) /$ $\mu(0)$ adult parasite-years. Therefore, over the course of the life-cycle each adult parasite-year results in $Q_{0}$ adult parasite-years in the next generation, where

$Q_{0}=\frac{q \lambda(0)}{(\rho+\beta H)} \frac{\beta H p(0)}{\mu(0)}$.

Both definitions of $Q_{0}$ are, of course, the same formula. This arises because equations (1)-(3) form an autonomous model, that is, one for which rates of transition between the stages in the life-cycle are independent of time. For nematode parasites transition rates between free-living stages often depend on the weather, and may be regarded as seasonal. Also, management interventions in farmed populations may cause discontinuous changes in parasite burdens. When time $(t)$ appears explicitly in the expressions for development rates, or parasite lifeexpectancy changes with time, then it is not so simple to define $Q_{0}$ in terms of the system parameters. The latter definition (in terms of parasiteyears) leads directly, in time-dependent cases, to a quantity with the correct threshold property. It may also be argued that the former is correct, but for nonautonomous systems a method of 'averaging' the number of parasites of the next stage produced over the year is required in order to provide a typical value. Correct averaging takes account of parasite life-expectancies, and once again the two definitions agree. This has been explained in more detail by Heesterbeek \& Roberts (1994).

The definition of $Q_{0}$ in terms of the changes in size of discrete generations recalls similar uses in ecology where, for example $R_{0}$ has been used as the net reproductive rate of a population (Southwood, 1978). This usage is consistent with ours only in the absence of density-dependent effects on population demography.

The expression for $Q_{0}$ defined by equation (4) is not unique in having the required threshold property, in fact $Q_{0}$ raised to any positive power also has the property. In the theory developed by Heesterbeek \& Roberts (1994), one of the ways to define and calculate $Q_{0}$ is to introduce a matrix $K$, derived directly from the model's equations in a simple way, and then to define $Q_{0}$ in terms of $\mathscr{R}(K)$, the dominant eigenvalue of $K$. More precisely, $\mathscr{R}(K)$ is the spectral radius of $K$, but for models of infectious diseases this is usually equal to the dominant eigenvalue (Diekmann et al. 1990). For the model defined by equations (1)-(3)

$K=\left(\begin{array}{cc}0 & \frac{q \lambda(0) H}{\rho+\beta H} \\ \frac{\beta p(0)}{\mu(0)} & 0\end{array}\right)$,

and the definition $Q_{0}=\mathscr{R}(K)^{2}$ leads directly back to equation (4). Any integer power of $\mathscr{R}(K)$ has the same threshold property. To justify our choice of $Q_{0}=\mathscr{R}(K)^{2}$ we point out that equations (1)-(3) are a simplification of a more complex system. For example, Grenfell, Smith \& Anderson (1987) presented a model for the population dynamics of Ostertagia ostertagi that consisted of 10 equations, 1 for egg production, 3 for the different larval stages on the herbage and 6 for the parasitic stages within the host. Equations (1 and 2) may be recovered by combining the first 4 and the last 6 respectively, and making some mathematical approximations based on time-scale arguments. If we had defined $Q_{0}=\mathscr{R}(K)$ then different values of $Q_{0}$ would be obtained for the 10-equation model and our 2-equation model. It would be unfortunate if a biologically defined quantity changed in value according to the degree of simplification of a mathematical model. Our definition, which is $Q_{0}=\mathscr{R}(K)^{k}$ where $k$ is the number of stages in the model life-cycle, overcomes this difficulty (see Heesterbeek \& Roberts, 1994).

In many situations the transition rates between different parts of the life-cycle are not independent of time. Roberts \& Grenfell (1991) investigated a scenario where farm management strategies were modelled by a periodic resetting of one or more state variables, and Roberts \& Grenfell (1992) analysed a model where the dynamics of the larval phase were seasonal and therefore time-dependent. The second situation requires equations (1) and (2) to be replaced with

$\frac{d L}{d t}=-(\rho(t)+\beta(t) H) L+q(t) \lambda(r) H A$

$\frac{d A}{d t}=\beta(t) p(r) L-\mu(r) A$,

where $\rho, \beta$ and $q$ are now functions of time $t$. A simple expression for $Q_{0}$ such as equation (4) can no longer be obtained. The number of parasites produced in the next stage as a result of a single parasite in the present stage is now a time-dependent quantity, and some mechanism is required to relate this to a typical parasite. With the realistic assumption that these time dependencies are periodic, Heesterbeek \& Roberts (1994) developed a sche:.. 
Table 1. Parameter values used in the examples

\begin{tabular}{|c|c|c|c|}
\hline Parameter & Value & Function & Definition \\
\hline$p(0)$ & $0 \cdot 65$ & $p(r)=p(0) \exp \left(-10^{-5} r\right)$ & Probability that an ingested larva develops into an adult parasite \\
\hline$q\left(=q_{0}\right)$ & $0 \cdot 5$ & $q(t)=q_{0}(1+\sin 2 \pi t)$ & Probability that an egg develops into an infective larva \\
\hline$H$ & $0 \cdot 0015 \mathrm{~m}^{-2}$ & & Host population density \\
\hline$\beta$ & $23733 \mathrm{yr}^{-1}$ & Constant & Rate at which larvae are eaten by host animals \\
\hline$\lambda(0)$ & $1678 \mathrm{yr}^{-1}$ & Constant & Mean rate at which an adult parasite produces eggs \\
\hline$\mu(0)$ & $6 \cdot 4 \mathrm{yr}^{-1}$ & Constant & Mortality rate of adult parasites \\
\hline$\rho$ & $356 \mathrm{yr}^{-1}$ & Constant & $\begin{array}{l}\text { Rate at which larvae are lost from the pasture, for reasons other } \\
\text { than being eaten by host animals }\end{array}$ \\
\hline$\sigma$ & $0 \cdot 01 \mathrm{yr}^{-1}$ & Constant & $\begin{array}{l}\text { Rate at which acquired immunity would decline in the absence of } \\
\text { larval challenge }\end{array}$ \\
\hline
\end{tabular}

whereby the matrix equivalent to $K$ (containing the instantaneous parasite-years in the next stage resulting from a single parasite-year in the present stage), could be averaged over a whole period, resulting in a matrix, $\mathscr{K}$, with $Q_{0}=\mathscr{R}(\mathscr{K})^{k}$. A similar argument formalizes the definition of $Q_{0}$ when state variables are periodically reset to model management practices. Although the construction of $\mathscr{K}$, and hence the definition of $Q_{0}$, make sound biological sense, they can no longer be calculated explicitly in terms of the system parameters, and must be found by numerical solution of the model.

Heesterbeek \& Roberts (1994) presented an alternative threshold quantity for the time-dependent problem that makes good pragmatic sense and is easier to calculate than $Q_{0}$. The procedure is as follows. First of all, solve equations (5) numerically over a single period (1 year) with initial conditions $L(0)=1, A(0)=0$. The resulting values of the variables at the end of the period are $L_{1}$ and $A_{1}$ say. Now repeat the procedure, with initial conditions $L(0)=0, A(0)=1$, obtaining $L_{2}$ and $A_{2}$ say. We have in effect defined a mapping $I \rightarrow E$, from the initial parasite population to the end-point population, where

$I=\left(\begin{array}{ll}1 & 0 \\ 0 & 1\end{array}\right)$ and $E=\left(\begin{array}{cc}L_{1} & L_{2} \\ A_{1} & A_{2}\end{array}\right)$.

The first and second columns of $E$ are the numbers of parasites in each stage after one period, resulting from a single initial larva and a single initial adult respectively. In Heesterbeek \& Roberts (1994) it is proved that $\mathscr{R}(E)<1$ if and only if $Q_{0}<1$, and hence $\mathscr{R}(E)$ may be used as a threshold quantity for non-autonomous (periodic) macroparasite models. It must be stressed that $\mathscr{R}(E)$ does not equal $Q_{0}$.

The numerical examples used throughout this paper are based on the parameters used by Roberts (1994b) to model the dynamics of Trichostrongylus colubriformis in lambs in New Zealand. The parameter values are given in Table 1 . For these parameter values $Q_{0}=7 \cdot 75$ for the autonomous model (parameters as in Table 1 , with $q=q_{0}$ ) as calculated from equation (4). A control measure based on chemotherapy may be modelled by replacing $\mu(0)$ with $\mu(0)+c$ ( $c$ is the increase in the rate of mortality due to control), and to use this mechanism to reduce $Q_{0}$ to 1 would require $c=43 \cdot 2$. For the autonomous model $\mathscr{R}(E)=6.52 \times 10^{16}$, and replacing $\mu(0)$ with $\mu(0)+c$ (as above) reduces this to 1 . Clearly, the entries in the matrix $E$ can get very large, but $\mathscr{R}(E)$ still has the correct threshold property. Note that $c$ is approximately equal to the number of successful treatments/year, so for these parameter values it is not practical to achieve eradication of the parasite population by chemotherapy.

For the seasonal model (parameters as in Table 1, with $\left.q=q_{0}(1+\sin 2 \pi t)\right)$ similar values of $\mathscr{R}(E)$ and $c$ were obtained (see Table 2). Roberts \& Grenfell (1991) modelled the annual replacement of stock on infected pasture by resetting $A$ (in equations 1 and 2, or 5 ) and $r$ (in equation 3 ) to zero every year. When this was incorporated in the model small reductions in $\mathscr{R}(E)$ and $c$ were again observed (see Table 2). Although resetting (i.e. stock replacement) dramatically changes the temporal pattern of parasite abundance, it does little to affect the control effort required to eradicate the parasite population. This is because the direct effect of resetting on $Q_{0}$ is through the removal of 1-year-old host animals, and hence their parasites. At this time the animals have lost the majority of their parasite burdens, and have acquired an immunity to reinfection.

The values of $Q_{0}$ for the seasonal models, and those with resetting, that are presented in Table 2 have been estimated from $\mathscr{R}(E)$ and $c$. This is because direct computation of $Q_{0}$ involves the inversion of a matrix, which for these parameter values is nearly singular, causing the numerical procedure to become unstable. $Q_{0}$ has therefore been approximated by $(\mu+c) / \mu$, where $c$ is the value that reduces $\mathscr{R}(E)$, and consequently $Q_{0}$, to 1 . It is therefore $Q_{0}$ for an equivalent (in some sense) autonomous model. 
Table 2. Values of the threshold parameters $\left(Q_{0}\right.$ and $\mathscr{R}(E))$ and the level of control effort $(c)$ required to reduce these to 1 , for the autonomous (equations 1-3) and seasonal (equations 3 and 5) models, with and without annual stock replacement

(Parameter values are as in Table 1 . * Indicates that $Q_{0}$ could not be evaluated numerically, but has been estimated from $\mathscr{R}(E)$ and $c$. See text for further explanation.)

\begin{tabular}{llll}
\hline Model & $Q_{0}$ & $\mathscr{R}(E)$ & $c$ \\
\hline Autonomous & $7 \cdot 75$ & $6.52 \times 10^{16}$ & 43.2 \\
Seasonal & $7 \cdot 35^{*}$ & $1.07 \times 10^{16}$ & 40.6 \\
Autonomous with replacement & $6.36^{*}$ & $6.19 \times 10^{15}$ & $40 \cdot 7$ \\
Seasonal with replacement & $5 \cdot 97^{*}$ & $1.00 \times 10^{15}$ & 38.2 \\
\hline \hline
\end{tabular}

\section{MULTIPLE HOST TYPES}

Consider first the situation where there are two different types of host. We are thinking here of different age-classes sharing the same grazing, different breeds or the introduction (to a flock or herd) of animals that have been selected for nematode resistance. Equations (1-3) are replaced with

$$
\begin{aligned}
\frac{d L}{d t}= & -\left(\rho+\beta_{1} H_{1}+\beta_{2} H_{2}\right) \\
& \times L+q\left(\lambda_{1}\left(r_{1}\right) H_{1} A_{1}+\lambda_{2}\left(r_{2}\right) H_{2} A_{2}\right) \\
\frac{d A_{1}}{d t}= & \beta_{1} p_{1}\left(r_{1}\right) L-\mu_{1}\left(r_{1}\right) A_{1} \\
\frac{d A_{2}}{d t}= & \beta_{2} p_{2}\left(r_{2}\right) L-\mu_{2}\left(r_{2}\right) A_{2} \\
\frac{d r_{1}}{d t}= & \beta_{1} L-\sigma_{1} r_{1} \\
\frac{d r_{2}}{d t}= & \beta_{2} L-\sigma_{2} r_{2},
\end{aligned}
$$

where the subscripts 1 and 2 refer to host types 1 and 2 respectively. The matrix $K$ is now defined by

$$
K=\left(\begin{array}{ccc}
0 & \frac{q \lambda_{1}(0) H_{1}}{\rho+\beta_{1} H_{1}+\beta_{2} H_{2}} \frac{q \lambda_{2}(0) H_{2}}{\rho+\beta_{1} H_{1}+\beta_{2} H_{2}} \\
\frac{\beta_{1} p_{1}(0)}{\mu_{1}(0)} & 0 & 0 \\
\frac{\beta_{2} p_{2}(0)}{\mu_{2}(0)} & 0 & 0
\end{array}\right)
$$

The entries in $K$ are determined as follows. The rate of production of stage- 1 parasites (larvae) by stage- 2 parasites (adults in host type 1 ) is $q \lambda_{1}(0) H_{1}$ per year, and their life-expectancy is $1 /\left(\rho+\beta_{1} H_{1}+\beta_{2} H_{2}\right)$ years. Hence $K_{12}$ is the number of stage- 1 years due to a stage- 2 year. The other entries are calculated in a similar manner.

By definition $Q_{0}=\mathscr{R}(K)^{2}=a_{1} Q_{0}^{(1)}+a_{2} Q_{0}^{(2)}$, where $Q_{0}^{(1)}$ and $Q_{0}^{(2)}$ are the values of $Q_{0}$ that would hold for host types 1 and 2 respectively if the other host type were absent (equation 4 with appropriate indexing). The factors $a_{1}=\left(\rho+\beta_{1} H_{1}\right) /\left(\rho+\beta_{1} H_{1}+\beta_{2} H_{2}\right)<1$ (similarly $a_{2}$ ) account for the reduced amount of larvae ingested by host type 1 due to competition by host type 2 .

\section{Example 1 : lambs and eques}

As an example of two different host types grazing together, consider a mixed flock of lambs and ewes (types 1 and 2 respectively). Although some component of the resistance of ewes to infection will be due to their past experience, we will model their increased resistance by taking $p_{2}(0)=0 \cdot 2$ (arbitrarily), with all other parameters the same for both types (see Table 1). Assuming the stock density for each host type to be $7 \cdot 5$ animals per hectare (to preserve $15 \mathrm{ha}^{-1}$ overall) we obtain $Q_{0}^{(1)}=4.06$ and $Q_{0}^{(2)}=1 \cdot 25$. Hence $Q_{0}=5 \cdot 07$, a reduction from $7 \cdot 75$ due to the replacement of half the flock with more resistant animals.

If chemotherapy were applied to host types 1 and 2 at rate $c_{1}$ and $c_{2}$ respectively, resulting in a basic reproduction quotient $Q_{c}$ for the parasite population under control, then

$Q_{c}=\frac{a_{1} \mu_{1}(0)}{\mu_{1}(0)+c_{1}} Q_{0}^{(1)}+\frac{a_{2} \mu_{2}(0)}{\mu_{2}(0)+c_{2}} Q_{0}^{(2)}$.

For example, if $c_{1}=c_{2}=4$ ( 4 effective treatments/ year for all host animals) then $Q_{c}=3 \cdot 12$. For a given total number of treatments $\left(c_{1}+c_{2}\right.$ fixed $)$ the minimum value of $Q_{c}$ is obtained when

$$
\frac{a_{1} \mu_{1}(0)}{\left(\mu_{1}(0)+c_{1}\right)^{2}} Q_{0}^{(1)}=\frac{a_{2} \mu_{2}(0)}{\left(\mu_{2}(0)+c_{2}\right)^{2}} Q_{0}^{(2)} \text {. }
$$

With our parameters, and the restriction $c_{1}+c_{2}=8$, this is achieved when $c_{1}=7$ and $c_{2}=1$, leading to $Q_{c}=2 \cdot 88$. The optimum strategy is therefore to apply the majority of treatments to the hosts that would, on their own, lead to the highest value of $Q_{0}$. The optimum values of $c_{1}$ and $c_{2}$ that result in $Q_{c}=1$, and hence lead to eradication of the parasite population are given in Table 3 , together with a summary of threshold parameters for seasonal models and models with replacement (and two hosts).

When the dynamics of the parasite populations under chemotherapy are examined separately for each host type, it is found that $a_{1} \mu_{1} Q_{0}^{(1)} /$ $\left(\mu_{1}+c_{1}\right)=2 \cdot 38$ and $a_{2} \mu_{2} Q_{0}^{(2)} /\left(\mu_{2}+c_{2}\right)=0.73$ when $c_{1}=c_{2}=4$. Hence the parasite population could not maintain itself in the ewe population at this host density and level of treatment, and the lamb population acts as a reservoir. However, when $c_{1}=7$ and $c_{2}=1$ the corresponding figures are 1.85 and 1.03 respectively, and the parasite population can just maintain itself within the ewe population. 
Table 3. Values of the threshold parameters $\left(Q_{0}\right.$ and $\mathscr{R}(E))$ and the level of control effort $(c)$ required to reduce these to 1 , for Example 1

\begin{tabular}{lllll}
\hline \hline & & $Q_{0}$ & $\mathscr{R}(E)$ & $c$ \\
\hline Autonomous & Lambs & 4.06 & & 32.14 \\
& Ewes & 1.25 & & 14.98 \\
& Overall & 5.07 & $3.03 \times 10^{10}$ & \\
\hline Seasonal & & $1.21 \times 10^{10}$ & \\
Autonomous with replacement & $2.07 \times 10^{9}$ & \\
Seasonal with replacement & $8.15 \times 10^{8}$ & \\
\hline \hline
\end{tabular}

\section{Example 2 : breeding for resistance}

Suppose parasite control were to be achieved by replacing a proportion of the host population with resistant animals. If, initially, we have $Q_{0}$ as defined by equation (4), and a proportion $s$ of the hosts were replaced with animals having a different value of $p(0)$, (hence $p_{1}(0)$ and $p_{2}(0)$ for host types 1 and 2 respectively) and all other parameters identical, then

$Q_{c}=\left(1-s+s \frac{p_{2}(0)}{p_{1}(0)}\right) Q_{0}$.

Using our example parameters (Table 1) for host type 1 , and assuming arbitrarily $p_{2}(0) / p_{1}(0)=0 \cdot 1$, then the smallest value of $s$ that renders the parasite population no longer viable $\left(Q_{c}<1\right)$ is 0.97 . This demonstrates that a minority of susceptible host animals can act as a reservoir of parasites for a majority of resistant animals. Even if $p_{2}(0)$ were equal to zero, we would have $s=1-1 / Q_{0}$, and the proportion of the flock that has to be replaced tends to 1 for large $Q_{0}$.

When stock replacement is included in the model, we find numerically that $Q_{c}=1$ when $s=0 \cdot 88$, which corresponds to $88 \%$ of the host animals being required to be resistant to achieve eradication of the parasite population, or ensure that the parasite population is no longer viable. The critical $s$ values for the corresponding seasonal model are equal to those of the autonomous model, to the nearest percentage point.

\section{MULTIPLE PARASITE TYPES}

In many ruminant grazing systems animals are infected not with a single nematode species, but with a community of them. This led Leathwick, Barlow \& Vlassoff (1992) to develop a model for nematodiasis in New Zealand lambs, rather than a model for a single parasite species. In the present paper we investigate the establishment threshold for a second parasite type (type is used to indicate either species or strain within a species) in the presence of a first, where there is some reciprocity in the immunity acquired by the host against the two types.

We assign subscripts 1 and 2 to the variables $L, A$, and $r$, parameters $q, \rho, \beta$, and $\sigma$, and functions $p, \lambda$ and $\mu$ in the obvious way, but $p, \lambda$ and $\mu$ may now depend on $r_{1}$ and $r_{2}$. The equations for the dynamics of the two parasite types are

$$
\begin{aligned}
\frac{d L_{1}}{d t} & =-\left(\rho_{1}+\beta_{1} H\right) L_{1}+q_{1} \lambda_{1}\left(r_{1}, r_{2}\right) H A_{1} \\
\frac{d A_{1}}{d t} & =\beta_{1} p_{1}\left(r_{1}, r_{2}\right) L_{1}-\mu_{1}\left(r_{1}, r_{2}\right) A_{1} \\
\frac{d r_{1}}{d t} & =\beta_{1} L_{1}-\sigma_{1} r_{1}
\end{aligned}
$$

with similar equations for type 2 . Assume now that type 1 is established, and in a (dynamic) equilibrium in the host population, and consider the threshold problem for type 2 . The small amplitude equations for parasite type 2 are

$$
\begin{aligned}
& \frac{d L_{2}}{d t}=-\left(\rho_{2}+\beta_{2} H\right) L_{2}+q_{1} \lambda_{2}\left(r_{1}, 0\right) H A_{2} \\
& \frac{d A_{2}}{d t}=\beta_{2} p_{2}\left(r_{1}, 0\right) L_{2}-\mu_{2}\left(r_{1}, 0\right) A_{2}
\end{aligned}
$$

where $r_{1}$ is calculated from equations (7) with $r_{2}=0$, or alternatively equations (1)-(3) with appropriate parameters for type 1 ; i.e. the solution of the equations for the dynamics of parasite type 1 with type 2 absent. If parasite type 1 is in a steady equilibrium, then we can write down $Q_{0}$ for type 2 directly,

$Q_{0}^{(2)}=\frac{q_{2} \lambda_{2}\left(r_{1}, 0\right) \beta_{2} H p_{2}\left(r_{1}, 0\right)}{\left(\rho_{2}+\beta_{2} H\right) \mu_{2}\left(r_{1}, 0\right)}$,

and parasite type 2 can invade if $Q_{0}^{(2)}>1$. Observe that replacing the parameter and function subscripts 2 with subscript 1 in the definition of $Q_{0}^{(2)}$ gives, not the formula for $Q_{0}^{(1)}\left(Q_{0}\right.$ for parasite type 1$)$, which requires $r_{1}=0$, but an effective reproduction quotient for parasite type $1\left(Q^{(1)}\right.$ say). As we have assumed this type to be in equilibrium, $Q^{(1)}=1$, which demonstrates that for parasite type 2 to invade it must have some fitness advantage over the established parasite population (of type 1), at the prevailing levels of acquired immunity $\left(r_{1}\right)$ in the host population. This assumes, of course, that parasite type 2 occupies exactly the same niche in the host population as parasite type 1 .

If we have annual stock replacement leading to periodic resetting, or seasonal development rates, equations (7) and (8) define a non-autonomous threshold problem that must be solved numerically. This may be done by solving equations (7) with $r_{2}=0$ for parasite type 1 , and then using $r_{1}$ so obtained in equations (8) to calculate $\mathscr{R}(E)$, the dominant eigenvalue of the matrix $E$.

\section{Example 3: two parasite types with reciprocal immunity}

Suppose that a chemotherapy programme were to 
reduce the abundance of parasite type 1 , and hence reduce $r_{1}$ to $r_{c}$. There is a concurrent change in the value of $Q_{0}$ for parasite type 2 to $Q_{c}^{(2)}$, where

$Q_{c}^{(2)}=\left(\frac{\lambda_{2}\left(r_{c}, 0\right)}{\lambda_{2}\left(r_{1}, 0\right)}\right)\left(\frac{p_{2}\left(r_{c}, 0\right)}{p_{2}\left(r_{1}, 0\right)}\right)\left(\frac{\mu_{2}\left(r_{1}, 0\right)}{\mu_{2}\left(r_{c}, 0\right)+c_{2}}\right) Q_{0}^{(2)}$.

The first two factors in the definition of $Q_{c}^{(2)}$ are greater than or equal to one, and $\mu_{2}\left(r_{1}, 0\right) \geqslant \mu_{2}\left(r_{c}, 0\right)$. If $c_{2}$ were small the effect of chemotherapy on parasite type 2 would be to increase $Q_{0}$, and the ability of that type to invade the host population. Note that if all parameter values for parasite types 1 and 2 are identical, $Q_{c}^{(2)}=1$, and parasite type 2 has no advantage over type 1 . This example will be discussed in the context of the development of parasite strains that are resistant to chemotherapy in the next section.

\section{THE INCORPORATION OF GENETICS IN THE MODEL}

Consider a nematode population with three genotypes (two homozygous and one heterozygous), arising from a simple genetical system. We label the two alleles $R$ and $S$, and subdivide the parasite population into $R R, R S$ and $S S$ strains. The equations for the dynamics of larvae of the $R R$ strain on pasture and adult parasites of the $R R$ strain within the host are

$$
\begin{aligned}
& \frac{d L_{R R}}{d t}=-(\rho+\beta H) L_{R R}+q \lambda(r) H \Lambda_{R R}\left(A_{R R}\right. \\
& \left.+A_{R S}+A_{S S}\right) \\
& \frac{d A_{R R}}{d t}=\beta p(r) L_{R R}-\mu(r) A_{R R},
\end{aligned}
$$

with similar equations for the $R S$ and $S S$ strains. The equation for acquired immunity is

$\frac{d r}{d t}=\beta\left(L_{R R}+L_{R S}+L_{S S}\right)-\sigma r$.

The functions $\Lambda_{R R}, \Lambda_{R S}$ and $\Lambda_{S S}$ are the proportions of eggs of each particular strain that are produced, as functions of the numbers of adult parasites of each strain present in the population. Hence

$\Lambda_{R R}=\frac{A_{R R}^{2}+A_{R R} A_{R S}+A_{R S}^{2} / 4}{\left(A_{R R}+A_{R S}+A_{S S}\right)^{2}}$

$\Lambda_{S S}=\frac{A_{S S}^{2}+A_{S S} A_{R S}+A_{R S}^{2} / 4}{\left(A_{R R}+A_{R S}+A_{S S}\right)^{2}}$

and

$$
\Lambda_{R S}=\frac{2 A_{R R} A_{S S}+A_{R R} A_{R S}+A_{R S} A_{S S}+A_{R S}^{2} / 2}{\left(A_{R R}+A_{R S}+A_{S S}\right)^{2}} .
$$

In deriving these relationships it has been assumed that parasite numbers are always sufficient for each female parasite to be able to find a mate. A similar development for the dynamics of nematode popu- lations of humans may be found in Anderson, May \& Gupta (1989), together with a discussion of the necessary corrections at low parasite density when this assumption is not satisfied.

Assume now that the parasite population is in a (dynamic) equilibrium with the ' $R$-gene' absent. Then ( $\left.L_{S S}, A_{S S}, r\right)$ obey equations (1)-(3), and we denote this solution by $(L, A, r)$. We construct the threshold property for the invasion of the ' $R$-gene' by considering small fluctuations in parasite population density about $(L, A, r)$. The equations for $L_{R R}$ and $A_{R R}$ are therefore

$$
\begin{aligned}
\frac{d L_{R R}}{d t} & =-(\rho+\beta H) L_{R R} \\
\frac{d A_{R R}}{d t} & =\beta p(r) L_{R R}-\mu(r) A_{R R} .
\end{aligned}
$$

In deriving these equations it has been recognized that $A_{R R}$ and $A_{R S}$ are both small quantities, and hence $A_{R R}$ is the sum of products of small quantities, and may be neglected. The solutions for $L_{R R}$ and $A_{R R}$ tend to zero over time, therefore the ability of the $R$ gene to invade the parasite population is determined by the dynamics of the heterozygous parasite population. The equations for $L_{R S}$ and $A_{R S}$ are (neglecting $A_{R R}$, which tends to zero)

$$
\begin{aligned}
& \frac{d L_{R S}}{d t}=-(\rho+\beta H) L_{R S}+q \lambda(r) H A_{R S} \\
& \frac{d A_{R S}}{d t}=\beta p(r) L_{R S}-\mu(r) A_{R S},
\end{aligned}
$$

which are equivalent to equations (8). The problem of whether the $R S$ strain can establish when the $S S$ strain is present may therefore be addressed in an identical manner to the analysis already presented for two parasite types. The relevant threshold quantity is

$Q_{0}^{(R)}=\frac{q \lambda(r) \beta H p(r)}{(\rho+\beta H) \mu(r)}$.

Once again we see that if the $R S$ strain has no advantage over the $S S$ strain, then by definition $Q_{0}^{(R)}=1$.

\section{Example 4 : the development of parasite strains resistant to chemotherapy}

Consider a parasite population in which the ' $S$-gene' predominates. If chemotherapy has been introduced, and the parasite population has attained a new equilibrium, then

$\frac{q \lambda\left(r_{c}\right) \beta H p\left(r_{c}\right)}{(\rho+\beta H)\left(\mu\left(r_{c}\right)+c\right)}=1$

where $c$ is the increase in parasite mortality due to chemotherapy, and $r_{c}$ is the value of $r$ under chemotherapy. Suppose now that the chemotherapy 
increases the mortality of the heterozygous $R S$ strain by an amount $c_{R}$, less than or equal to $c$. It is then immediate that

$Q_{0}^{(R)}=\frac{q \lambda\left(r_{c}\right) \beta H p\left(r_{c}\right)}{(\rho+\beta H)\left(\mu\left(r_{c}\right)+c_{R}\right)}=\frac{\mu\left(r_{c}\right)+c}{\mu\left(r_{c}\right)+c_{R}}$.

This expression is analogous to that for $Q_{c}^{(2)}$ in Example 3, and provides a measure of the ability of the $R$-gene to establish. Using the parameters in Table 1 , and letting $c=4$ and $c_{R}=0$ for example, we obtain $Q_{0}^{(R)}=1 \cdot 63$. Alternatively, solving equations (1)-(3) with $\mu$ replaced with $\mu+c$ for the $S S$ strain, and using the value of $r$ so obtained in the solution of equation (10) leads to $\mathscr{R}(E)=49 \cdot 3$ in the absence of annual host replacement, and $\mathscr{R}(E)=49 \cdot 2$ with host replacement. Note that $Q_{0}$ for the $S S$ strain during chemotherapy is, for this example, $Q_{c}=4 \cdot 77$. The control measure is, therefore, insufficient to eradicate the $S S$ strain $\left(Q_{c}>1\right)$, but confers an advantage on the resistant strains $\left(Q_{0}^{(R)}>1\right)$.

\section{Example 5 : resistance to chemotherapy with two host types}

The equations for the dynamics of a parasite population with two host types (equations 6), have a steady state solution when $Q_{0}=a_{1} Q_{0}^{(1)}+a_{2} Q_{0}^{(2)}>1$, where $a_{1}$ and $a_{2}$ are as defined previously. The steady state values of $r_{1}$ and $r_{2}$ may be determined from $a_{1} Q^{(1)}\left(r_{1}\right)+a_{2} Q^{(2)}\left(r_{2}\right)=1$, where

$Q^{(1)}\left(r_{1}\right)=\frac{q \lambda_{1}\left(r_{1}\right) \beta_{1} H_{1} p_{1}\left(r_{1}\right)}{\left(\rho+\beta_{1} H_{1}\right) \mu_{1}\left(r_{1}\right)}$

with a similar expression for $Q^{(2)}\left(r_{2}\right)$. If a chemotherapy programme that consists of increasing the mortality of parasites in host types one and two by $c_{1}$ and $c_{2}$ respectively, reduces $r_{1}$ to $r_{c}$ at steady state, then $r_{c}$ may be determined from

$$
\begin{aligned}
& \frac{a_{1} \mu_{1}\left(r_{c}\right)}{\mu_{1}\left(r_{c}\right)+c_{1}} Q^{(1)}\left(r_{c}\right)+\frac{a_{2} \mu_{2}\left(r_{c}^{\prime}\right)}{\mu_{2}\left(r_{2}^{\prime}\right)+c_{2}} Q^{(2)}\left(r_{c}^{\prime}\right)=1 \\
& r_{c}^{\prime}=\frac{\beta_{2} \sigma_{1}}{\beta_{1} \sigma_{2}} r_{c} .
\end{aligned}
$$

Similar reasoning to that in Example 4 yields

$$
Q_{0}^{(R)}=\frac{a_{1} \mu_{1}\left(r_{c}\right)}{\mu_{1}\left(r_{c}\right)+c_{R 1}} Q^{(1)}\left(r_{c}\right)+\frac{a_{2} \mu_{2}\left(r_{c}^{\prime}\right)}{\mu_{2}\left(r_{c}^{\prime}\right)+c_{R 2}} Q^{(2)}\left(r_{c}^{\prime}\right)
$$

In Example 1 it was assumed that the epidemiological parameters of both parasites were as in Table 1 (particularly $\mu_{1}=\mu_{2}=\mu$ ), apart from $p_{1}(0)=0.65$ and $p_{2}(0)=0 \cdot 2$. In Example $5 c_{R}$ was assumed to be zero. Repeating these assumptions, and using $c_{1}=c_{2}=c$ and $H_{1}=H_{2}=H / 2$ (hence $a_{1}=a_{2}$ ) leads to $r_{c}=r_{c}^{\prime}, a \mu\left(Q^{(1)}+Q^{(2)}\right) /(\mu+c)=1$ and therefore $Q_{0}^{(R)}=(\mu+c) / \mu$, as in Example 4. Furthermore, $Q^{(1)}(r) / p_{1}(0)=Q^{(2)}(r) / p_{2}(0)$ for all $r$ and $\mu$ is independent of $r$, so similar arguments to those used in Example 1 show that when a fixed number of treatments $\left(c_{1}+c_{2}\right)$ are available $Q_{0}^{(R)}$ is minimized when

$\frac{a_{1} \mu_{1} p_{1}(0)}{\left(\mu_{1}+c_{1}\right)^{2}}=\frac{a_{2} \mu_{2} p_{2}(0)}{\left(\mu_{2}+c_{2}\right)^{2}}$

As in Example 1, when the total number of treatments is fixed at 8 the optimum strategy with our parameters is $c_{1}=7, c_{2}=1$. The strategy to minimize the development of resistance in nematodes is the same as the strategy to optimally control the nematode population: apply the majority of treatments to the most susceptible host type.

\section{DISCUSSION}

The basic reproduction ratio has been the cornerstone of models for infectious diseases since the Dahlem conference in 1982 (Anderson \& May, 1982). During this time the concept has been borrowed frequently for application to models of helminth parasites (for example Anderson \& May (1991) and other references cited earlier). These uses were not incorrect, but when parasite life-cycles with transmission or mortality rates that vary with time (leading to seasonal models), or production systems incorporating regular farmer intervention (leading to models with resetting) are considered, more formal definitions are required (Heesterbeek \& Roberts, 1994). We have seen in this paper how a formal definition aids in the clarification of concepts in parasite control when heterogeneity in either host or parasite complicate the life-cycle.

Two distinct threshold quantities have been discussed. The tentatively named basic reproduction quotient $\left(Q_{0}\right)$ for parasite populations has two alternative definitions, which coincide for autonomous models. The definitions, in terms of either parasites or parasite-years in the next generation are equal if the former is correctly averaged over a time period. The value of $Q_{0}$ is easy to calculate for an autonomous model, and may be explicitly expressed for autonomous models with resetting. However, in the latter case numerical instability prevented its calculation when our example parameter values were incorporated. For seasonal models $Q_{0}$ must be calculated numerically, and similar difficulties may occur. For the examples in the present paper it was found that the easily calculated value of $Q_{0}$ for the autonomous system without resetting gave a useful guide to its values for more complicated models.

The quantity $\mathscr{R}(E)$ has a precise mathematical definition, and is easier to calculate than $Q_{0}$ for all but the autonomous model with no resetting, but has less biological meaning. In fact, the use of $\mathscr{R}(E)$ as a threshold quantity has a circular feeling to it, along the lines 'if a population increases in size, then it increases in size'. We have confirmed that, in all our examples, model control interventions that reduced $Q_{0}$ to one also reduced $\mathscr{R}(E)$ to one. Despite its lack 
of intuitive appeal, $\mathscr{R}(E)$ is often a useful quantity for determining the long-term dynamics of parasite populations.

Even though these threshold quantities must often be calculated numerically, their use offers considerable advantage over the determination of longterm parasite population dynamics by carrying out a series of simulations. Apart from the usual arguments concerning the generality of qualitative results in opposition to the specificity of results from simulations (see Mollison, Isham \& Grenfell, 1994), $Q_{0}$ and $\mathscr{R}(E)$ are calculated from the linearization of the model equations. This means that for many examples the non-linearities (i.e. the precise nature of the dynamics of acquired immunity) need not be determined, and the amount of data required to analyse the model is reduced. It is acknowledged that the model used to illustrate these threshold quantities is highly simplified. It does, however, contain the main features of the nematode life-cycle in farmed ruminants and could therefore be applied to problems involving parasites in sheep, cattle or goats. Simulation models must be tailored to a particular system and invariably contain many more parameters (see for example Grenfell et al. (1987); Barnes \& Dobson (1990) and Smith \& Grenfell (1994) and references therein). The simple model used here has been shown to display dynamical behaviour that is qualitatively correct (Roberts, $1994 b$; Roberts \& Grenfell, 1991; 1992).

Equations (6) were used to model the influence of two distinct types of host on parasite population dynamics, and the method could easily be extended to more than two. By different host types we mean distinct groups of animals that have different interactions with the parasite population, but are both able to be infected by it. It was found that $Q_{0}$ for the parasite population could be expressed as a linear combination of the two values of $Q_{0}$ that would apply if each host type were present in isolation. It was also found that if chemotherapy were to be applied as a control or eradication strategy, it should be directed for preference to the host type with the largest $Q_{0}$. In Example 1, if a total of 8 treatments were available then 7 should be applied to the lambs and one to the ewes. In Example 2 the replacement of host animals with others more resistant to infection by parasites was investigated. It was found that a high proportion of host animals need to be replaced for this to be an effective parasite eradication strategy in the absence of other control measures.

The dynamics of more than one parasite type in a farmed host population has been discussed. This problem has previously been examined in wild animal populations (Dobson, 1985; Roberts \& Dobson, 1994), and experimentally for farmed animals where there may be a shared acquired immunity between two parasite species (for example, prior infection of lambs with $O$. circumcincta may provide protection against subsequent infection with T. colubriformis (Dobson, Barnes \& Windon, 1992)). The model demonstrates that for a second species of parasite to invade where a first is already established it must have some advantage, and that the advantage may be a direct consequence of a control intervention. These ideas were built upon with reference to the development of parasite strains resistant to chemotherapy.

A model for the dynamics of a parasite population with an emerging gene for resistance was proposed. It was found that the criterion for the development of resistance is determined by the transmission and mortality parameters of the susceptible strain and the heterozygous strain. This is because for small densities of the ' $R$-gene' the probability that a parasite with the gene is able to find one of the opposite sex with the same gene is low, and the $R R$ strain cannot invade until the $R S$ strain has established. It was shown in Example 4 how the use of chemotherapy as a control measure gives the $R S$ strain the advantage required to invade the population. In Example 5 the use of chemotherapy where there are two host types and a gene for enhanced parasite resistance to chemotherapy was discussed. It was found that $Q_{0}^{(R)}$, the threshold quantity for the development of resistance, is minimized when chemotherapy is applied in preference to the host type with greatest individual $Q_{0}$.

Finally, in all of these examples the use of chemotherapy has been modelled by increasing parasite mortality by a value $c$. Although approximately true when a large number of treatments are applied, this would be more appropriate for a sustained release capsule. Individual treatments could be modelled by the resetting of state variables, in a similar manner to that used for farm management practice above (but without changing the value of $r$ ). The timing of interventions would then be important in determining the threshold criteria. This will be discussed in a future paper.

This work was conceived while the authors took part in the programme on epidemic models at the Isaac Newton Institute for Mathematical Sciences, University of Cambridge. The authors are grateful to the organizers of the programme, and to the institute, for the opportunity to participate. M. G.R. also received financial assistance from a Trimble Agricultural Research Fellowship and MSDAgVet Ltd and an extended period of leave from the New Zealand Pastoral Agriculture Research Institute Ltd, J.A.P.H. was financially supported by the Netherlands Organization for Scientific Research, NWO; the Royal Society and SERC Grant GR G59981. Stewart Bisset made many helpful comments on an earlier draft of the paper.

\section{REFERENCES}

Anderson, R. M. (1982). The Population Dynamics of Infectious Diseases: Theory and Applications. New York: Chapman \& Hall. 
ANDERson, R. M. \& MAY, R. M. (1982). The Population Biology of Infectious Diseases. Berlin: Springer-Verlag. ANDERSON, R. M. \& MAY, R. M. (1991). Infectious Diseases of Humans: Dynamics and Control. Oxford: Oxford University Press.

ANDERSON, R. M., MAY, R. M. \& GUPTA, S. (1989). Nonlinear phenomena in host-parasite interactions. Parasitology 99, S59-S79.

BARNES, E. H. \& DOBSON, R. J. (1990). Population dynamics of Trichostrongylus colubriformis in sheep: a computer model to simulate grazing systems and the evolution of anthelmintic resistance. International fournal for Parasitology 20, 823-31.

DiEkMANN, O., heEsterbeeK, J. A. P. \& METZ, J. A. J. (1990). The definition and computation of the basic reproduction ratio $R_{0}$ in models of infectious diseases in heterogeneous populations. Fournal of Mathematical Biology 28, 365-82.

DiEKMANN, O., HEESTERBEeK, J. A. P. \& METZ, J. A. J. (1994). The legacy of Kermack and McKendrick. In Epidemic Models, their Structure and Relation to Data (ed. Mollison, D.) Cambridge: Cambridge University Press (In the Press.)

DOBSon, A. P. (1985). The population dynamics of competition between parasites. Parasitology 91 , $317-47$.

DOBSON, A. P. \& GRENFELL, B. T. (1994). Ecology of Infectious Diseases in Natural Populations. Cambridge: Cambridge University Press (In the Press.)

DOBSON, R. J., BARNES, E. H. \& WINDON, R. G. (1992). Population dynamics of Trichostrongylus colubriformis and Ostertagia circumcincta in single and concurrent infections. International Fournal for Parasitology 22, 997-1004

GRENFELL, B. T., SMITH, G. \& ANDERSON, R. M. (1987). A mathematical model of the population biology of Ostertagia ostertagi in calves and yearlings. Parasitology 95, 389-406.

heesterbeek, J. A. P. (1992). $R_{0}$. Ph.D. thesis. University of Leiden.

HeEsterbeek, J. A. P. \& Roberts, M. G. (1994). Threshold properties for helminth infections. Fournal of Mathematical Biology (In the Press.)

KERMACK, W.. O. \& MCKENDRICK, A. G. (1927).

Contributions to the mathematical theory of epidemics - 1. Proceedings of the Royal Society 115A, 700-21. Reprinted as Bulletin of Mathematical Biology 53, 33-55 (1991).

LEATHWICK, D. M., BARLOW, N. D. \& VLASSOFF, A. (1992).
A model for nematodiasis in New Zealand lambs. International Fournal for Parasitology 22, 789-99. Mollison, D. (1994). Epidemic Models, their Structure and Relation to Data. Cambridge: Cambridge University Press (In the Press.)

MOLLISON, D., ISHAM, v. \& GRENFELL, B. T. (1994). Epidemics: models and data. Fournal of the Royal Statistical Society A 157, 115-49.

ROBERTS, M. G. (1994a). Discussion section of Mollison, Isham \& Grenfell (1994), p. 136. Epidemics: models and data. Fournal of the Royal Statistical Society $\mathbf{A}$ 157, 115-49.

ROBERTS, M. G. $(1994 b)$. The population dynamics of nematode infections of ruminants. In Proceedings of the Third International Conference on Mathematical Population Dynamics, University of Pau, 1-5 Fune 1992, (ed. Kimmel, M.), Winnipeg: Wuerz Publishing (In the Press.)

ROBERTS, M. G. \& DOBSON, A. P. (1994). The population dynamics of communities of parasitic helminths. Mathematical Biosciences (in the Press.)

RoBERTS, M. G. \& GRENFELL, B. T. (1991). The population dynamics of nematode infections of ruminants : periodic perturbations as a model for management. IMA Yournal of Mathematics Applied in Medicine and Biology 8, 83-93.

ROBERTS, M. G. \& GRENFELL, B. T. (1992). The population dynamics of nematode infections of ruminants: the effect of seasonality in the free-living stages. IMA Fournal of Mathematics Applied in Medicine and Biology 9, 2941.

ROBERTS, M. G., LAWSON, J. R. \& GEMMELL, M. A. (1987). Population dynamics in echinococcosis and cysticercosis: Mathematical model of the life-cycles of Taenia hydatigena and T. ovis. Parasitology 94, 181-97.

ROBERTS, M. G., SMITH, G. \& GRENFELL, B. T. (1994). Mathematical models for macroparasites of wildlife. In Ecology of Infectious Diseases in Natural Populations, (ed. Dobson, A. P. \& Grenfell, B. T.), Cambridge: Cambridge University Press (In the Press.)

SCOTT, M. E. \& SMITH, G. (1994). Parasitic and Infectious Diseases: Epidemiology and Ecology. San Diego: Academic Press.

SMITH, G. \& GRENFELL, B. T. (1994). Modelling of parasitic populations: gastrointestinal nematode models. Veterinary Parasitology 54, 127-34. southwood, T. R. E. (1978). Ecological Methods. 2nd Edn, New York: Wiley. 